\title{
Lysine Regulation of Penicillin Biosynthesis in Low-producing and Industrial Strains of Penicillium chrysogenum
}

\author{
By JOSE M. LUENGO, GLORIA REVILLA, \\ JULIO R. VILLANUEVA AND JUAN F. MARTÍN \\ Departamento de Microbiología, Facultades de Ciencias y de Farmacia, \\ Universidad de Salamanca, Salamanca, Spain
}

(Received 22 January 1979; revised 2 May 1979)

The inhibitory effect of L-lysine on penicillin biosynthesis by Penicillium chrysogenum has been compared in a low-producing strain (Wis. 54-1255) and a high-producing strain (ASP-78). Lysine inhibited total penicillin synthesis to a similar extent in both strains. However, in the high-producing strain the onset of penicillin synthesis occurred even at a high lysine concentration, whereas in the low-producing strain lysine had to be depleted before penicillin production commenced.

\section{INTRODUCTION}

It is well established that lysine inhibits the biosynthesis of penicillin by Penicillium chrysogenum (Demain, 1957; Masurekar \& Demain, 1972). Since both lysine and penicillin are end-products of a branched pathway in which 2-aminoadipic acid is the branch point (Abraham, 1977), it has been proposed that lysine inhibition of penicillin synthesis is due to feedback regulation (Demain \& Masurekar, 1974; Demain, 1974). Studies in vivo suggest that the site of the lysine effect on the biosynthesis of penicillin is homocitrate synthase, the first enzyme of the lysine biosynthetic pathway (Demain \& Masurekar, 1974).

The industrial relevance of the regulatory effect of lysine on the biosynthesis of penicillin has not been established. It is possible that industrial mutant strains have lost sensitivity to lysine inhibition of penicillin synthesis during selection. Moreover, the lysine content of some media may not reach inhibitory concentrations for penicillin synthesis. There is increasing evidence which suggests that industrial strains are overproducers of antibiotics because they have lost regulatory mechanisms which exist in wild-type strains (Martín, 1978). Acetohydroxyacid synthase, the first enzyme of the valine biosynthetic pathway, is less sensitive to valine inhibition in industrial strains of $P$. chrysogenum (Goulden \& Chattaway, 1969).

The present study compares the regulatory effect of lysine on the biosynthesis of penicillin in a low-producing strain of $P$. chrysogenum with that in a high-producing strain.

\section{METHODS}

Microbial strains. A low-producing strain of Penicillium chrysogenum Wis. 54-1255 (a member of the Wisconsin family) was provided by Dr A. L. Demain (M.I.T., Cambridge, Mass., U.S.A.) and an old highproducing strain, AS-P-78, was a gift from Antibióticos, S.A. (León, Spain). Strain AS-P-78 is a prototroph but grows very slowly on minimal medium. Production of penicillin in shake flasks by this strain is about $5000 \mu \mathrm{g} \mathrm{ml}^{-1}$ (about 8000 units $\mathrm{ml}^{-1}$ ), but production in the fermenter may reach 12000 units $\mathrm{ml}^{-1}$. Strains were kept lyophilyzed or in liquid $\mathrm{N}_{2}$ (gas phase).

Culture media and growth conditions. The inoculum was grown in medium containing ( $\left.\mathrm{g}^{-1}\right)$ : corn-steep liquor, 20; distiller solubles, 20; sucrose, 20; $\mathrm{CaCO}_{3}$, 5. The $\mathrm{pH}$ was adjusted to $5 \cdot 7$ before sterilization. 
The defined production medium contained $\left(\mathrm{g} \mathrm{I}^{-1}\right)$ : glucose, 10; sucrose, 10; lactose, 30; citric acid, 10; acetic acid, 2.5; potassium phenylacetate, 2 ; ethylamine, $3 ;\left(\mathrm{NH}_{4}\right)_{2} \mathrm{SO}_{4}, 5 ; \mathrm{KH}_{2} \mathrm{PO}_{4}, 1 ; \mathrm{FeSO}_{4} .7 \mathrm{H}_{2} \mathrm{O}, 0.05$; $\mathrm{MgSO}_{4} .7 \mathrm{H}_{2} \mathrm{O}, 0.5 ; \mathrm{ZnSO}_{4} .7 \mathrm{H}_{2} \mathrm{O}, 0.01 ; \mathrm{CuSO}_{4} .5 \mathrm{H}_{2} \mathrm{O}, 0.01 ; \mathrm{MnSO}_{4} .4 \mathrm{H}_{2} \mathrm{O}, 0.01 ; \mathrm{CoSO}_{4} .7 \mathrm{H}_{2} \mathrm{O}, 0.005 ;$ $\mathrm{NaCl}, 0.001 ; \mathrm{CaCO}_{3}, 10$. Additional sucrose was added daily between 3 and $6 \mathrm{~d}$ at final concentrations of $10 \mathrm{~g} \mathrm{I}^{-1}$ (J. L. Fernández, Antibióticos, S. A., personal communication). The $\mathrm{pH}$ of this medium was adjusted to $6 \cdot 2$ before sterilization ( $\mathrm{pH}$ after sterilization $8 \cdot 0$ ).

The complex production medium contained $\left(\mathrm{g}^{-1}\right)$ : corn-steep solids, 35; lactose, 25; potassium phenylacetate, $2.5 ; \mathrm{MgSO}_{4} .7 \mathrm{H}_{2} \mathrm{O}, 3 ; \mathrm{KH}_{2} \mathrm{PO}_{4}, 7$; corn oil, $2.5 ; \mathrm{CaCO}_{3}, 10$. Defined and complex media were sterilized at $121^{\circ} \mathrm{C}$ for 20 and $30 \mathrm{~min}$, respectively.

Seed media $(50 \mathrm{ml})$ in $250 \mathrm{~m} .1$ triple-baffled flasks were inoculated with a conidial suspension (final density of $10^{7}$ conidia ml-1). After growing for $40 \mathrm{~h}$ at $25^{\circ} \mathrm{C}$ in a rotary shaker at $250 \mathrm{rev} . \mathrm{min}^{-1}$, mycelia were collected, washed by centrifugation and suspended in sterile water $(10 \mathrm{ml})$. This mycelial suspension $(2.5 \mathrm{ml})$ was used to inoculate complex or defined production medium $(50 \mathrm{ml})$ in $500 \mathrm{ml}$ flasks. Incubation for penicillin production was carried out at $25^{\circ} \mathrm{C}$ for $5 \mathrm{~d}$.

Determination of penicillin. Culture broths were centrifuged at $1800 \mathrm{~g}$ for $10 \mathrm{~min}$. The supernatant fluid was centrifuged again for $5 \mathrm{~min}$ at $1500 \mathrm{~g}$ and the clear supernatants were used for the routine assay of penicillin with Bacillus subtilis ATCC 6633 as test strain (Cooper, 1972). Penicillin was sometimes also determined colorimetrically with the hydroxylamine reagent (Bcxer \& Everett, 1949) to confirm the results.

Lysine uptake. L-[U-14 $\left.{ }^{14}\right] l y$ sine monohydrochloride $\left(270 \mathrm{mCi} \mathrm{mmol}^{-1}\right)$ and $\mathrm{DL}-\left[4,5(n)-{ }^{3} \mathrm{H}\right] \mathrm{lysine}$ monohydrochloride (10 Ci mmol-1) were purchased from The Radiochemical Centre, Amersham. Radioactively labelled L-lysine (17000 c.p.m. $\mathrm{ml}^{-1}$ ) was added at inoculation. The resulting specific activity of lysine in the broth was calculated by allowing for the amount of unlabelled lysine added to the different flasks. Samples of the broth $(1 \mathrm{ml})$ were filtered rapidly through glass-fibre filters, dried and their radioactivity was measured in a Packard Tricarb 3320 scintillation counter. Samples $(50 \mu \mathrm{l})$ of filtered broth were chromatographed on silica gel $G$ thin-layer plates using the solvent system butan-1-ol/acetic acid/water $(8: 2: 2$, by vol.) and the lysine spots were cut out and counted.

\section{RESULTS}

Penicillin production by low- and high-producing strains in defined medium

In control cultures of strain Wis. 54-1255 without lysine (Fig. $1 a$ ), production of penicillin started after a large increase in cell dry weight and glucose exhaustion. Addition of $10 \mathrm{~mm}$ lysine (Fig. $1 \mathrm{~b}$ ) delayed the onset of penicillin synthesis until after $48 \mathrm{~h}$ growth but the rate of penicillin synthesis thereafter was greater, reaching the same concentration as in the control after $72 \mathrm{~h}$. Addition of $10 \mathrm{~mm}$-lysine did not affect the growth rate or the final dry weight of the culture.

Cultures supplemented with $50 \mathrm{~mm}$-lysine (Fig. 1c) showed a delay in the onset of penicillin synthesis and a large reduction in the production rate thereafter. This concentration of lysine did not affect the growth of the culture, nor was the $\mathrm{pH}$ affected by the addition of lysine.

The high-producing strain AS-P-78 showed a slightly lower growth rate and a much higher rate of penicillin production (Fig. 1d). Cultures of strain AS-P-78 supplemented with 10 mM-lysine showed the same inhibitory effect on penicillin synthesis as strain Wis. 54-1255 and a similar delay in the onset of rapid penicillin synthesis (Fig. $1 e$ ). Supplementation of strain AS-P-78 with $50 \mathrm{~mm}$-lysine produced a similar effect, although the final yield of penicillin was not very different from that of control cultures (Fig. 1f).

\section{Penicillin production by low- and high-producing strains in complex medium}

The complex medium differed from lysine-free synthetic medium in having a significant amino acid content in the form of both free amino acids and proteins.

Penicillin production by control cultures of strain Wis. 54-1255 reached $800 \mu \mathrm{g} \mathrm{ml}^{-1}$ in complex medium, but addition of $10 \mathrm{~mm}$-lysine at inoculation delayed the onset of penicillin biosynthesis and decreased the final yield of penicillin (Fig. $2 a$ ). If $10 \mathrm{~mm}$-lysine was added daily, synthesis of penicillin was inhibited by $80 \%$. The addition of $50 \mathrm{~mm}$-lysine at the time of inoculation resulted in a considerable delay and reduction in the rate of biosynthesis of 


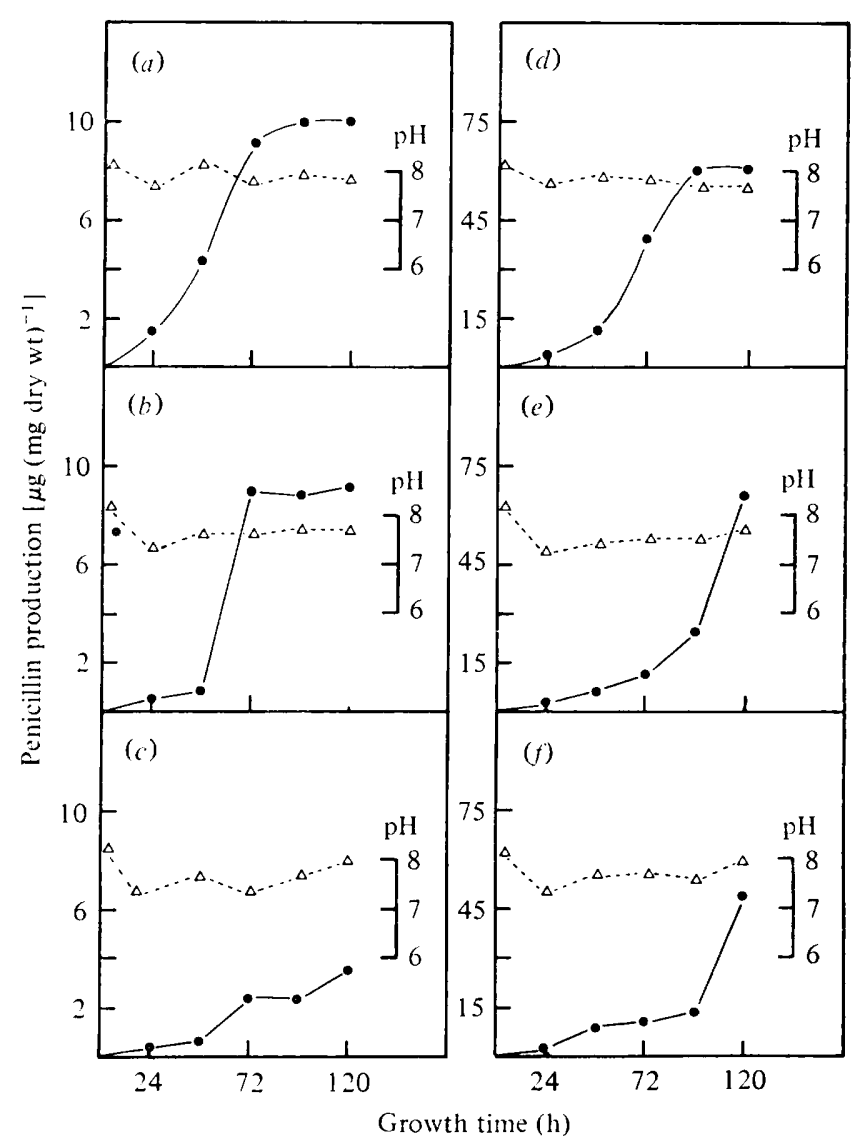

Fig. 1. Penicillin production $(\odot)$ and $\mathrm{pH}$ variation $(\triangle)$ during growth of Penicillium chrysogenum strains Wis. 54-1255 $(a, b, c)$ and AS-P-78 $(d, e, f)$ in synthetic medium: control $(a, d)$; supplemented with $10 \mathrm{~mm}$-lysine $(b, e)$ or $50 \mathrm{~mm}$-lysine $(c, f)$.

penicillin. The production of penicillin was completely suppressed when 50 mm-lysine was added daily (Fig. $2 b$ ).

The high-producing strain AS-P-78 behaved similarly except that the addition of either 10 or 50 mm-lysine did not delay the onset of penicillin synthesis but simply decreased the rate of synthesis (Fig. $2 c$ ). Daily addition of $10 \mathrm{~mm}$-lysine decreased the final yield of penicillin considerably, and daily addition of $50 \mathrm{~mm}$-lysine completely suppressed the biosynthesis of penicillin (Fig. $2 d$ ). Lysine addition did not affect the growth rate or the growth yield in either of the two media.

\section{Lysine consumption during penicillin production}

To check whether the delay in the onset of penicillin synthesis observed in strain Wis. 541255 at high concentrations of lysine was due to the longer time required for depletion of lysine in the broth, we measured the consumption of labelled lysine by this strain in complex medium. Residual lysine was measured in the broth after t.l.c. separation. When the culture was supplemented with 10 mm-lysine, $90 \%$ had been utilized before 48 h (Fig. $3 a$ ). Synthesis of penicillin was derepressed at this time following lysine utilization.

When the culture was supplemented with 50 mM-lysine, utilization proceeded linearly for about $48 \mathrm{~h}$ until $85 \%$ had been utilized. This extensive incorporation of lysine did not result in increased dry weight, which suggests that under normal growth conditions (excess 


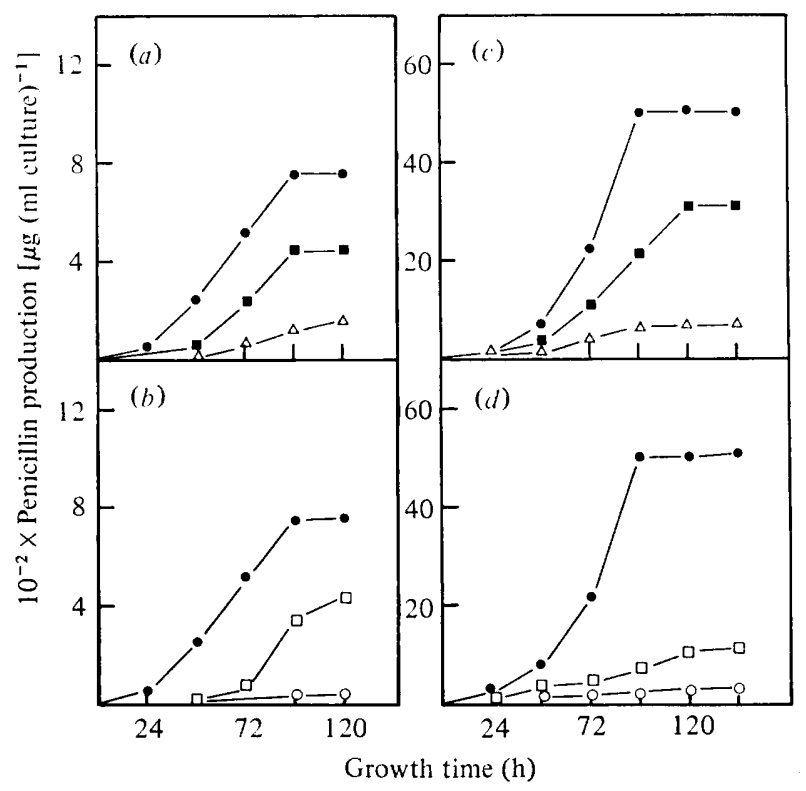

Fig. 2. Penicillin production by Penicillium chrysogenum strains Wis. 54-1255 $(a, b)$ and AS-P-78 $(c, d)$ in complex medium: control $(\bullet)$; supplemented with $10 \mathrm{~mm}$-lysine ( $\mathbf{\square})$ or $50 \mathrm{~mm}$-lysine $(\square)$ at the time of inoculation; supplemented with $10 \mathrm{~mm}$-lysine $(\triangle)$ or $50 \mathrm{~mm}$-lysine $(O)$ daily.

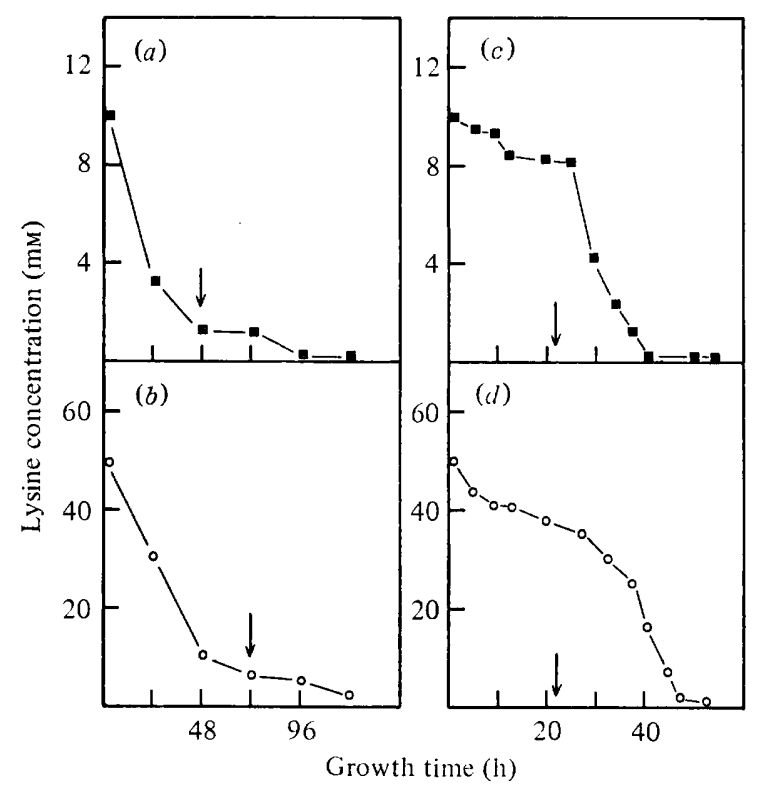

Fig. 3. Lysine utilization by Penicillium chrysogenum strains Wis. 54-1255 $(a, b)$ and AS-P-78 $(c, d)$ in complex medium: supplemented with $10 \mathrm{~mm}$-lysine $(\square)$ or $50 \mathrm{~mm}$-lysine $(O)$. The onset of penicillin synthesis occurred at the times indicated by the arrows.

of lactose as carbon source) it did not contribute significantly to the formation of biomass. The onset of penicillin synthesis in cultures of Wis. 54-1255 supplemented with 50 mMlysine was delayed until most of the lysine had been utilized, which occurred at about $72 \mathrm{~h}$ (Fig. 3b). 
The incorporation of lysine by strain AS-P-78 proceeded slowly in the first day of growth in cultures supplemented with either 10 or 50 mM-lysine. Thereafter, lysine was utilized more rapidly (Fig. $3 c, d$ ). Synthesis of penicillin in this strain started at about $24 \mathrm{~h}$, when the level of residual lysine was still quite high. The onset of penicillin synthesis in AS-P-78 cultures supplemented with $50 \mathrm{~mm}$-lysine occurred at residual lysine concentrations as high as $35 \mathrm{~mm}$ (Fig. $3 d$ ). This suggested that the onset of penicillin synthesis in strain AS-P-78 was less sensitive to lysine than in strain Wis. 54-1255.

\section{DISCUSSION}

The production of penicillin by the high-producing strain AS-P-78 is almost as sensitive to lysine regulation as that by the low-producing strain (Fig. 1). This raises the possibility of obtaining mutants insensitive to lysine regulation, which might be overproducers of penicillin. The onset of penicillin production by strain Wis. 54-1255 was delayed until the lysine concentration of the broth decreased below non-inhibitory levels (Figs 1, 2, 3). However, the onset of penicillin production by the high-producing strain AS-P-78 in the complex medium was not delayed. The addition of lysine in this case resulted in a decreased rate of penicillin synthesis, but production started at the same time (Fig. $2 c, d$ ). Depletion of $50 \mathrm{~mm}$-lysine by strain AS-P-78 before $24 \mathrm{~h}$ of growth did not occur (Fig. $3 c, d$ ); we conclude that in complex media, the high-producing strain is still able to produce penicillin in the presence of lysine, although at a reduced rate. This may indicate that the onset of biosynthesis of penicillin by the high-producing strain in complex medium is less sensitive to lysine regulation. In other words, selection of this high-producing strain through the empirical industrial procedures has not completely removed the mechanisms of lysine regulation of penicillin biosynthesis, but it appears that the onset of penicillin synthesis is less sensitive to the presence of lysine.

Daily addition of lysine to either strain (Fig. $2 b, d$ ) almost completely suppressed penicillin production. We have evidence that this is due to repression and inhibition of the enzymes synthesizing penicillin (J. M. Luengo \& J. F. Martín, unpublished observations).

We thank Antibióticos, S.A. (León, Spain) for financial support and the gift of strain AS-P-78. We are greatly indebted to Drs J. García-Jimeno, F. Holguera and J. L. Fernández for their continual interest in this research project.

\section{REFERENCES}

Abraham, E. P. (1977). $\beta$-Lactam antibiotics and related substances. Japanese Journal of Antibiotics 30 Supplement, S-21-S-26.

Boxer, G. E. \& EveretT, P. M. (1949). Colorimetric determination of benzylpenicillin. Analytical Chemistry 21, 670-673.

COOPER, K. E. (1972). The theory of antibiotic diffusion zones. In Analytical Microbiology, vol. 2, pp. 13-30. Edited by F. Kavanagh. London \& New York: Academic Press.

DemaIN, A. L. (1957). Inhibition of penicillin formation by lysine. Archives of Biochemistry and Biophysics 67, 244-245.

Demain, A. L. (1974). Biochemistry of penicillin and cephalosporin fermentations. Lloydia 37, 147-166.

Demain, A. L. \& Masurekar, P. S. (1974). Lysine inhibition of in vivo homocitrate synthesis in
Penicillium chrysogenum. Journal of General Microbiology 82, 143-151.

Goulden, S. A. \& Chattaway, F. W. (1969). Endproduct control of acetohydroxyacid synthetase by valine in Penicillium chrysogenum Q176 and a high penicillin-yielding mutant. Journal of General Microbiology 59, 111-118.

Martín, J.F. (1978). Manipulation of gene expression in development of antibiotic production. In Antibiotics and Other Secondary Metabolites: Biosynthesis and Production, pp. 19-37. Edited by R. Hutter, T. Leisinger, J. Nuesch \& W. Wehrli. London \& New York: Academic Press.

Masurekar, P. S. \& Demain, A. L. (1972). Lysine control of penicillin biosynthesis. Canadian Journal of Microbiology 18, 1045-1048. 